\title{
Building a model for encouraging help-seeking for depression: a qualitative study in a Chinese society
}

\author{
Alison KY Hui ${ }^{1 \dagger}$, Paul WC Wong ${ }^{2 \dagger}$ and King-wa Fu ${ }^{1 *}$
}

\begin{abstract}
Background: Clinical depression has been increasingly prevalent in international health statistics but people are often found to be reluctant to seek help when they encounter depression. However, there is no general theory to explain how personal, social and cultural factors affect an individual's help-seeking intention, nor to guide the design of preventive programmes for such intention once needed.
\end{abstract}

Methods: Drawing on the theory of planned behavior, we deployed the illness narrative approach and interviewed 18 participants in Hong Kong.

Results: With the diverse results we gathered from the interviews, a behavioral model was built to conceptualize the interplays of various factors in shaping one's help-seeking intention and behavior for depression. Participants appeared to have a limited view of treatment options and had diverse views of the symptoms of depression, both of which profoundly affected their motivation to seek help.

Conclusions: The role of family and friends and a holistic approach to mental health education were found to be particularly important for encouraging help-seeking behavior in future campaigns concerning depression.

\section{Background}

About 121 million people around the world are currently affected by depression (WHO 2011). It is projected to become a major leading contributor to the global burden of disease (Murray et al. 2012). However, only less than $25 \%$ of the individuals with depression are being treated effectively, partly due to barriers like the lack of treatment resources, social stigma and a limited number of trained professionals to reach the fast growing quantity of people in need of services (WHO 2011). Also, individual's low help-seeking incentive was found to be influenced by social, cultural and personal factors, which often include stigma attached to the malady and a lack of knowledge about depression (Barney et al. 2006).

In view of the rising concern revolving around mental health, media have been used as platforms to implement widespread psycho-educational materials and public

\footnotetext{
* Correspondence: kwfu@hku.hk

${ }^{\dagger}$ Equal contributors

'Journalism and Media Studies Centre, The University of Hong Kong, Hong Kong, China

Full list of author information is available at the end of the article
}

communication campaigns to de-stigmatize mental illness and educate the public about mental health and treatment options (Wahl 2003). Media campaigns have been found to be helpful in increasing exposure to depression and acceptance of it among the public (Paykel et al. 1998; Hegerl et al. 2003). However, these campaigns are often disseminated in special settings where those individuals in need are already present: school, patient groups or specific social group settings which may not reflect the general public's perception of depression and their help-seeking attitude (Halgin et al. 1987; Good and Wood 1995; Reiling 2002). Also, the relative-effectiveness and cost-effectiveness of these media campaigns were found to be inconstant depending on the campaign's duration, thematic approach and strategic practice (Elder et al. 2004; Grausgruber et al. 2009). On the other hand, researchers suggested that media campaign can effectively cultivate positive health behavioral changes among the mass public when the policies, programmes and services needed for the changes are made available and accessible (Wakefield et al. 2010).

This present study draws on data from an initial stage of a multiple-phase, mixed-methods research project which 
aims to develop a public health communication media campaign to encourage help-seeking for depression. It also aims to formulate a theory-driven model that guides the campaign development and incorporates contextualized and culturally relevant media content. As an initial part of a mixed-methods study, this paper reports on the qualitative formative research aimed at conceptualizing the basis of the theoretical framework to explain the formation of the public's view of depression and help-seeking behavior.

\section{How to encourage help-seeking?}

With media as platforms for mental health campaigns, the use of effective campaign content and material is also essential to help make the campaigns influential. Previous research found that better knowledge of mental illness is associated with higher help-seeking intention (Burns and Rapee 2006; Jorm 2000). Researchers notice that perceptions, attitudes and belief systems are essential factors to encourage help-seeking about an illness (Blay et al. 2008). Mental health literacy, which involves both knowledge and belief about mental illness, is found to greatly affect an individual's preferences and expectations regarding help-seeking behaviors and the prescribed treatments (Jorm et al. 2000a; Jorm et al. 1997). Another frequent area of interest in considering a mental health campaign content is to minimize the negative effect of stigma on help-seeking in depression (Ben-Porath 2002; Han et al. 2006). Common types of social stigma attached to depression include negative connotations, suggesting that a patient may be weak or violent (Barney et al. 2006). Another type of stigma is self-stigma, which refers to the beliefs of individuals who have formed negative views of self by incorporating how they think others would view them and their behavior relating to their illnesses (Griffiths et al. 2011a). Researchers find that self-stigma is likely to be a key factor in determining whether a person would seek help or turn to self-reliance when dealing with depression (Barney et al. 2006).

Previous studies have identified several personal factors that can influence an individual's help-seeking behavior concerning depression. They include inefficient monitoring of depression symptoms or a failure to adequately identify the condition and its early signs and symptoms (Cabassa 2007; Sherwood et al. 2007); belief in effectiveness of treatment (Roness et al. 2005a); perceived stigma and self-stigma accompanying depression, its treatment and help-seeking behavior (Barney et al. 2009).

Although the content of existing depression campaigns ranges diversely from anti-stigmatization to educating about general mental health knowledge to changing the public's attitudes or perceptions, very few are theoretically guided by a clear conceptual framework. The underlying mechanisms that enable a particular campaign to work and how it can change public attitudes toward help-seeking remain unclear. Rather than promoting mental health care usage, it is essential and more effective to model how these contributing factors are related systematically and what roles they play to influence an individual's attitude and belief toward help-seeking for depression. With such a model, future health campaigns can be better targeted in relation to these factors and specifically designed to encourage help-seeking behavior related to depression.

\section{Theory of planned behavior}

This study is a part of a four-stage research that the major aim is to create an online media campaign to encourage help-seeking incentive for depression. The first stage is to adopt the theory of planned behavior (TPB) as framework to lay out a pathway to study how individuals come to decide to seek help or not (Ajzen 1991). As a formative stage of the research, this study relied on the theory and research cycle which involved both inductive and deductive approach which allow theories, hypotheses, observations and empirical generalizations to inform one another (Wallace 1971). Such study would help inform the online media campaign strategy and content that was conducted in the later stages. Based on Ajzen's (1991) definitions, attitude consists of one's beliefs toward the behavior; a subjective norm involves one's perception of how others would view certain behaviors; and perceived behavioral control involves one's own feeling of self-efficacy toward conducting the behavior (Ajzen 1991). The TPB illustrates that an individual's attitude, perceived norms and perceived behavioral control can strongly determine that individual's intention and actual help-seeking behavior (Ajzen 1991). Instead of just encouraging the use of mental health services, we hope that this model can help to understand how social, personal and other factors can affect people's help-seeking behavior regarding depression. By understanding that, suitable measures and media messages can be designed and targeted to deal with the influence of these factors in order to help change the individual's attitude and behavior for help-seeking.

The TPB is one of the central theories used for public communication campaign strategies, processes and implementation (Rice and Atkin 2013).

\section{Identifying cultural factors through illness narrative}

Culture shapes our view, expression and interpretation of the symptoms and the treatment of depression (Kleinman 2004; Salloum and Mezzich 2009). Public conception and expression of depression varies across cultures (Kleinman et al. 1986). For example, Chinese villagers understood depression as excessive anger or worry and was usually diagnosed as neurasthenia, while people with the same symptoms would have been diagnosed as experiencing depression in Western culture (Kleinman et al. 1986). 
Chinese rural people expressed depressive symptoms as somatic or bodily complaints while Nigerians described them as "ants keep creeping in parts of my brain" (Kleinman et al. 1986; Ebigbo 1982). In a cross-cultural study, Australian Chinese is found to conceive depressive mood as stress while local Australians tend to see the same symptoms as the signs of depression, suggesting that each community has its own distinctive understanding of culture and illness, which in turn determines one's acceptance, help-seeking attitudes and behavior regarding depression (Chan 2007). Therefore it is essential to study the influence of culture in understanding an individual's view of depression and come up with more diverse and appropriate treatment and interventions that are specific for various cultures (Kleinman et al. 1986; Chan 2007).

Given that culture has a great influence on an individual's understanding of illness, preference to treatment, help-seeking behavior and intention, Kleinman (1988) suggests the use of the illness narrative as a strategy to allow an individual's explanation for his contextual understanding about the illness. The illness narrative approach allows individuals to formulate their own explanation models of how they name the illness they experience (Salloum and Mezzich 2009). This approach enables researchers to examine how various factors shape and contribute to the interviewee's view of depression and help-seeking. Using this approach, the understanding of cultural factors can greatly help media campaigners design culturally-sensitive and more effective health interventions that encourage help-seeking behavior (Salloum and Mezzich 2009).

Existing studies of ways to increase understanding of depression and help-seeking have primarily been conducted in Western communities but not in Chinese settings (Lee et al. 2007; Ajzen 1986). It is essential to look into encouraging help-seeking in depression as public health communication and preventive measure in Chinese societies, specifically identifying the role of cultural factors in creating help-seeking incentives and understanding the ways to reinforce such incentives and behavior. This research focuses on young Chinese adults because this group is known to be relatively more reluctant to seek professional help (Barney et al. 2006; Roness et al. 2005b).

Drawing on the above review, we aim to lay out a behavioral model to guide the development of public intervention for encouraging help-seeking for depression on the basis of the TPB and the use of the illness narrative approach. This model will guide the campaign material to be developed in later stages.

Two research questions are devised as follow:

1) How to develop a multifaceted framework to model the factors that can contribute to one's help-seeking attitude or behavior concerning depression?
2) How do various cultural factors affect one's help-seeking attitude or behavior and how can the identified factors be incorporated into the model developed by Research Question 1?

\section{Methods}

\section{Research setting}

This research took place in Hong Kong. As a cosmopolitan city in southern China, Hong Kong's rapid socioeconomic and demographic changes have led to an increasing rate of mental illnesses in the population especially in recent decades (Lee 1999). Scholars have attributed the rising rates to crowded living space, and to the stressful and competitive social environment of the city (Cheung et al. 1998). Other literature from the Western world also suggests that depression is seen to be related to one's success and failure in an individualistic culture, and that self-blame is common in more sociocentric and cosmopolitan cultures (Salloum and Mezzich 2009). One study finds that less than $20 \%$ of people with mentally illnesses in Hong Kong receive mental health services (Mo and Mak 2008).

\section{Protocol development}

As mentioned previously, culturally relevant information is needed in order to better understand depression, particularly in the setting of Hong Kong. We conducted semi-structured interviews to collect the culturally relevant information on how Hong Kong Chinese perceive depression. The semi-structured interviews were designed with reference to the McGill Illness Narrative Interview (MINI). MINI is structured as a story-telling approach to allow interviewees to express their views on an illness through their own narratives and explanatory models (Groleau et al. 2006). This interview model consists of five sections that include initial illness narrative, prototype narrative, explanatory model narrative, services and response to treatment and impact on the interviewee's life (Groleau et al. 2006). This approach to the narrative interview helps to elicit the person's actual and contextual understanding of an illness through his own narration and one's explanation of the help from different factors including friends, family and treatments, based on related experiential knowledge (Groleau et al. 2006). MINI was originally designed for patient interviews but we have adapted and extended it also for nonpatient interviewees. The questions in the interviews were also constructed with reference to the theory of planned behavior, which could infer people's attitude, perceived norms and self-efficacy about depression as mentioned (Ajzen 1991). The protocol used is provided in Appendix 1. The ethical aspects of this protocol were approved by the Human Research Ethics Committee for Non-Clinical Faculties, at the University of Hong Kong. 


\section{Data collection}

The duration of each qualitative interview in this study was about one to two hours. Three pilot interviews were carried out in the early stages of the study in order to inform and identify new questions that needed attention in this study. A total of 18 participants (Table 1) were obtained through purposive sampling from Hong Kong residents through the connections of authors (Patton 2002). They shared the common experience of Hong Kong culture and were invited according to their various background including age and occupation. The sampling stopped after the 18th interview due to saturation of response. These interviewees included mostly non-patients and three participants who had suffered from depression to speak from both knowledge and experience. The inclusion of participants who have and have not experienced depression is to fulfil the target of developing the public health communication and preventive intervention for the general public. Participants who have experienced depression were not explicitly excluded since the purpose of the study was to explore the general population's view on depression and help-seeking, including those with previous experience of depression and their secondary help-seeking intention. Their results were generally also analyzed together for this formative purpose instead of a specific comparative purpose. The inclusion of participants who have experienced depression helped to include a broader view of the picture and include factor that can influence ex-patient's help-seeking

Table 1 Profile of participants

\begin{tabular}{|c|c|c|c|}
\hline No. & Gender (F/M) & Age range & Occupation \\
\hline 1 & $\mathrm{~F}$ & $20-25$ & Lawyer \\
\hline 2 & $\mathrm{~F}$ & $20-25$ & Retail \\
\hline 3 & $\mathrm{~F}$ & $20-25$ & Disciplined service \\
\hline 4 & $\mathrm{~F}$ & $20-25$ & Fresh graduate \\
\hline 5 & F & $20-25$ & Speech therapist \\
\hline 6 & $\mathrm{~F}$ & $26-30$ & Retail \\
\hline 7 & $\mathrm{~F}$ & $20-25$ & Service industry \\
\hline 8 & $M$ & $35-40$ & Businessman \\
\hline 9 & $\mathrm{~F}$ & $20-25$ & Nurse \\
\hline 10 & M & $30-35$ & Information technology \\
\hline 11 & M & $20-25$ & Medical Doctor \\
\hline 12 & $\mathrm{~F}$ & $25-30$ & Retail \\
\hline 13 & $M$ & $20-25$ & Postgraduate student \\
\hline 14 & $M$ & $30-35$ & Businessman \\
\hline 15 & $\mathrm{~F}$ & $40-45$ & Teacher \\
\hline 16 & $\mathrm{~F}$ & $40-45$ & Air Hostess \\
\hline 17 & $\mathrm{~F}$ & $20-25$ & Student \\
\hline 18 & $M$ & $20-25$ & Student \\
\hline
\end{tabular}

incentive. The ratio and number of participants who have and have not experienced depression was not prearranged but was instead gained through purposive sampling in which the process stops when data saturation is reached. The participants comprised individuals with different age groups, gender, backgrounds, and occupations.

The interviewees provided a wide range of views on depression, help-seeking and how they came to think in such a way. These interview excerpts were gathered into a massive pool of views in order to conceptualize how to understand people's beliefs about depression. The first author of this paper conducted all of the interviews.

\section{Data analysis}

We deployed a theory-building approach with the TPB as framework but allowed essential variables like cultural factors and participants' own narrative to formulate and emerge with inductive emergence of theoretical constructs through data collection (Glaser and Strauss 1967; Eisenhardt 1989). On the other hand, the TPB was used as a framework to organize and categorize the theoretical ideas. The interview excerpts were then coded with the QSR International's NVivo 2 qualitative data analysis software, which helped to identity categories of views. Because the relationship between attitude and behavior is very complex, constant comparison was made between the data and the TPB model to keep the analysis systematic, culturally-sensitive and informative. Based on that comparison and other references, a model was then built to conceptualize an individual's help-seeking decision. The first author first transcribed and analyzed the original texts and then identified a draft of categories and themes from the data. Based on the draft, the first and the third authors revised the draft iteratively to generate the final list of themes and categories reported as follows. The categories were initially coded by the first author and then examined by the third author who is the principle investigator of the project. If disagreement occurred, both authors met, discussed, resolved the problems, and consequently consented to the final themes used in this study. This process helped address the issue of coding reliability. The second author provided clinical advice to the modifications.

\section{Results}

Views that emerged from the interviews were categorized into five major themes, including 1) attitude; 2) subjective norm; 3) perceived behavioral control; 4) actual behavioral control, and 5) treatment experience and consequent help-seeking attitude. The first three themes are the three main categories in the TPB model, along with 'actual behavioral control' in the decision process (Ajzen 1991). All themes are summarized into a diagram (Figure 1) to reflect their relationships. 


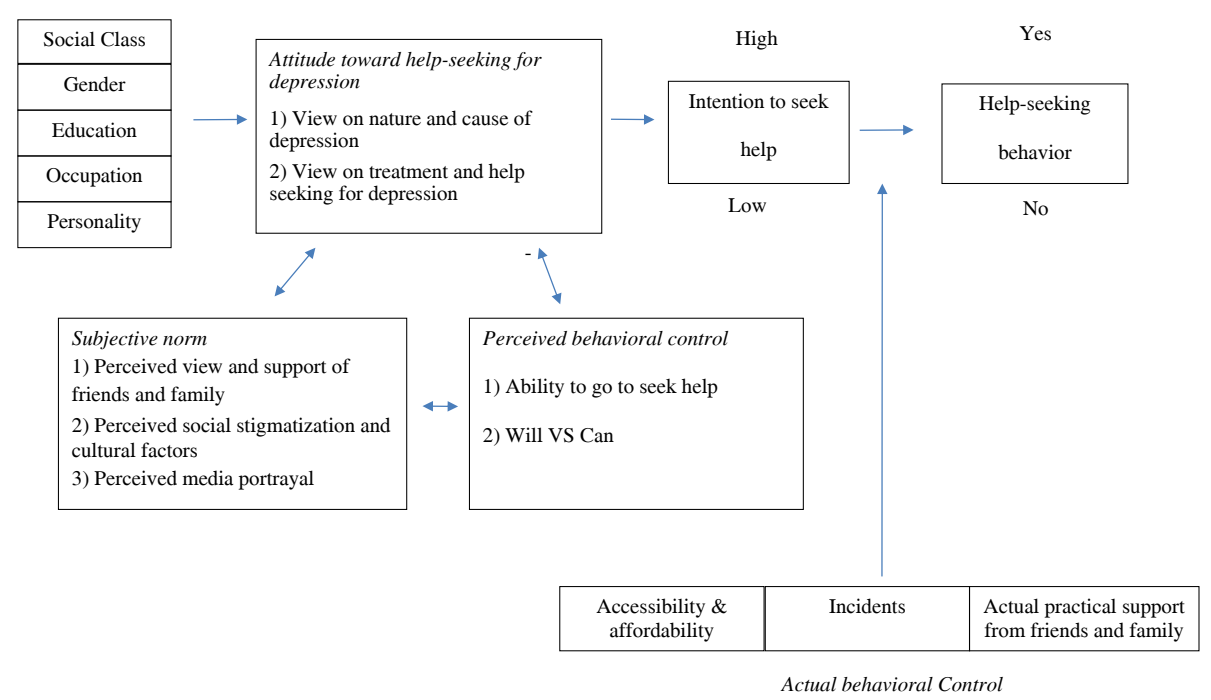

Figure 1 Factors that influence an individual's help-seeking behavior derived from the study results (Categories in Italic are formed deductively with reference to Ajzen, 1991).

\section{Attitude}

Participants expressed their attitude toward help-seeking for depression through questions that ask for their views on depression, causes of depression and treatment that shaped their help-seeking intention.

\section{View of the nature and causes of depression}

Participants generally recognized depression as a prolonged and more serious version of being unhappy that might lead to suicide. But they held diverse views on whether depression is an illness and such views influence their beliefs about the necessity of help-seeking. Participants who saw depression as an illness, either biological or physical, suggested professional help-seeking if one suffers from depression. This view links with the understanding that an illness like depression needs to be treated or that the treatment should not be delayed. Those participants who did not view depression as an illness also regarded it as a short term phenomenon and tended to have a lower intention to help-seeking. They were also inclined to see depression as a temporary emotional state which would fade away eventually and thus treatment may not be necessary.

"To heal it (depression), you have to 'untie the knot' in his heart. Depression is about psychological issues... Indeed I do not know the purpose of medication. I do not think depression can be settled by medication. It is not biological, but psychological." (Participant 6)

Along the same line, participants' understanding of the cause of depression seemed to reflect what measures they would take to deal with depression. Participants who believed the cause to be an external trigger or a factor only specific to individuals who are pessimistic or reticent prefer to receive non-pharmacological management, such as "talking it out" or psychological treatment. In the same regard, these participants did not prefer to receive treatment because they thought that depression was mostly related to one's own perspective and mentality. On the other hand, those who believed in biological causes, for example an imbalance in brain chemicals, tended to prefer psychiatric help and medical treatments.

\section{View regarding treatments and help-seeking for depression}

The individual's perception of treatment also has a significant impact on whether one plans to seek help and the type of treatment to consult. The treatments most often mentioned by participants are: talking with family and friends, counseling services or psychological interventions offered by a clinical psychologist or social worker, and pharmacological treatment by psychiatrist. Participants were inclined to have a presumed pathway to the timing, preference and steps to approach different treatment options according to the stage of being depressed and the severity of the illness. Many participants opted for psychological treatment at the initial stage and medication when the depressive episodes became severe and prolonged.

"I will go for a clinical psychologist first. I would try to refrain from a psychiatrist. I think if it is treated in time, you shouldn't need to consult a psychiatrist." (Participant 3) 
"I will drag until when I get manic and uncontrollable, or when I start to have unhealthy desires wanting to kill myself, then I would deal with it." (Participant 7)

Participants also strongly expressed their preference toward seeking help from friends or family at the first instance. Other participants specifically laid out the time frame they would follow to seek different treatments:

"When I realize that I am really unhappy, I would tell my family about it as the first step. Then after half a year if I remain like that, I will go to a clinical psychologist. If I am still drowning in the problems a year after, I will need drug." (Participant 1)

As for the treatment preference, pharmacological and psychological treatments were the two main options mentioned by the participants. Psychological treatments was seen by some participants to be a more acceptable and effective. In contrast, the effectiveness and the sideeffects of drugs concerned a number of participants. Outcome expectancy of the treatments was also a key factor to determine their treatment choices or help-seeking incentives as they thought some treatment might not lead to relevant, efficient or helpful outcomes. Their decisions about treatment were also strongly linked to their perception that causes of depression, as mentioned, included biological or psychological ingredients.

However, when our participants were asked about the treatment options that they knew of, most could only recall pharmacological and psychological treatments. Many of them were unaware of other self-help treatment options, such as exercise or St. John's Wort (Jorm et al. 2006). They were also unsure of the distinction between the treatments that a psychiatrist or a clinical psychologist could provide. Some also thought that they could recover from depression by themselves over time. The term "Psychological Doctor" (“心理醫生” in Chinese) is a common term used in Hong Kong to name both psychiatrists and clinical psychologists. These indicated that the scope of their knowledge of depression and help-seeking might be too narrow, which may have discouraged help-seeking behaviors.

As for their view on help-seeking, participants had various opinions about whether it was necessary to seek help based on their above mentioned views on the causes and nature of depression and the effectiveness of treatment. A few participants expressed worry over the stigma attached to help-seeking that might prevent individuals with depression from seeking professional help. Although their comments were mostly positive toward help-seeking, many of them had concerns over the high cost of medical and psychological treatment and the long waiting times for these treatments. Many participants thought that professional help was approachable if they went to a nearby public hospital or clinic at the first instance. But some said they would seek a recommendation from friends or church members for professional help. Another approach that has arisen in the last few years is to search for relevant help-seeking information online.

\section{Subjective norm}

An individual's perceived norm may contribute to whether one would have the incentive to seek help or not when experiencing depression. Previous studies suggest that social stigma would be one of the most influential perceived factors to prevent individuals from going to seek help (Griffiths et al. 2011a; Ajzen 1991). However, in our interviews, perceived support or disapproval by friends and family seemed to be more influential than social stigma.

\section{Perceived view and support from friends and family}

Many participants referred to the importance of having support of family and friends if they decided to seek help. This support indeed relates to both the perceived level of support and the actual practical help that the individual might receive. This kind of practical help, known as actual behavioral control, is explained in the last part of the diagram.

"I will ask them to bring me to the doctor. I trust my family's decision. I definitely won't go on my own. I need them to make booking for me and go with me that is the only way I can accept [treatment]." (Participant 5) "I will discuss with my family first to see whether I really have this illness. After that, I will seek help from doctors that I know or search online for a clinical psychologist."

(Participant 2)

They also mentioned that the prospect of making family members worry could also be a factor in whether someone would consider seeking help or not. Indeed, this specific influence of worrying family and friend varies greatly among individuals. If the bonding is not strong or the family is not supportive, family influence could be negative. Therefore, some participants simply thought that family's support was not an important factor in their decision to seek help.

"I think they (family) would support me to seek help. But I think I would insist to go to the clinical psychologist (even if the family doesn't support) since I could at least find out if I am sick or not. The opinion of family members is not professional advice. Even they think it is not necessary to see a doctor, I don't think it is absolutely correct." (Participant 2) 
Although the influence of family and friends might vary greatly, they are still important figures because they can easily spot emotional differences for the individual. For example, many participants mentioned that they could easily spot depressive episodes in their own close family and friends. Several participants indicated that they could immediately realize emotional changes by people around them but it would take much longer for them to observe and realize these changes in themselves. In short, the family and friends may be important gatekeepers with greater ability to spot changes in people than those individuals possess. Therefore, earlier identification of changes in depressive symptoms by family and friends would help one deal with those changes it at an earlier stage. This might indicate that a help-seeking campaign might not only increase self-awareness or the likelihood of self-initiated help but also encourage efforts to get help making use of the close connection of family and friends.

\section{Perceived social stigmatization and cultural factors}

Many participants had a feeling that there was less stigmatization toward depression currently and said they believed that the public was more open to talking about depression compared to seeing it as a serious mental illness and madness in the past. But many participants still said that they would be afraid to admit the condition, or to let others know if they had experienced depression. They said that people with depression were usually seen to be problematic, fragile or incapable. The linkage to weakness seemed to be more profound in Hong Kong, which is a stressful society that emphasizes personal achievement. Several participants pointed out that it is hard to differentiate stress from the clinical depressive state. They thought that feeling stress was very common in a competitive society like Hong Kong. Although depression is less attached to stigma than violence nowadays, participants mentioned that depression was still problematic. Participants thought they would be scared and uncertain about how to help a person with depression in that such actions might provoke his emotion.

"No matter it is depression or bipolar, people will link it to mental illness...people would worry that they would be physically attacked when faced with a person with depression or bipolar. Even I should control myself not to think that way, but I still feel very scared in the beginning." (Participant 5)

With the perceived stigma that participants think they would face, it is understandable that these factors could prevent them from help-seeking when they experience depression. However, throughout our interviews, participants seemed to be relatively more concerned about the efficiency of the treatments rather than the stigma attached to depression.

\section{Perceived media portrayal}

The media play an important role in shaping how the public view depression. The portrayal of depression in the media also informs the public about how depression is seen in the eyes of other individuals in the society. Participants said that the media coverage of depression that they have seen was mostly about suicide. Most media reports portrayed the consequences of depression, and arguably the reports might encourage participants to seek help in order to avoid such a sad ending. However, media coverage could also be a barrier that makes participants think depression is distant from them if the reports convinced them they should not expect such a bad scenario to happen to people around them.

\section{Perceived behavioral control Ability to seek help}

Perceived behavioral control is a very tricky variable in this study, in part because participants can hardly predict their own emotional state when experiencing depression. Therefore that assessment is just the participant's own prediction based on beliefs about the state and symptoms of depression. Several participants stated that they do not believe they have the rational ability to seek help in that situation. They took this position because they thought that depression would take away their ability to fulfil daily activity and they would not even be able to seek help.

\section{Will vs. Can}

Another essential point is that participants worried that they might not even realize they are sick when they were to experience depression. They were concerned that even if they think help-seeking is important right now, they still would not go to seek help because they would not realize that they had depression when they were in a depressive state. They worried that the influence of depression might not allow them to be aware of their own state, or would lead them to reject their own depressive state, which would in turn prevent them from proceeding to seek help.

"I don't think I will realize. I need to rely on the people near me to recognize it and tell me that I am not doing okay." (Participant 11)

\section{Actual behavioral control}

Actual behavioral control refers to realistic factors that would directly influence the behavior despite other attitudinal reasons (Ajzen 1991). The main factors that emerge from the interviews include accessibility, affordability, 
effects of previous incidents and actual help from family and friends.

As mentioned previously, an individual's view of accessibility and affordability might influence his attitude toward help-seeking. However, when it comes to the actual situation, individuals might not be able to seek help due to difficulties in accessibility and affordability even if they wished to seek help right at that moment. These factors can constitute effective barriers for individuals who might not be able to afford and find the treatments despite their intention to seek help.

Participants who have experienced depression have also mentioned the occurrence of various incidents that would encourage them to seek help. These include being diagnosed and helped by general practitioners during consultations or treatment for some other disease. Other incidents may include seeing leaflets as part of campaigns that help them realize that they might have been going through depressive episodes. Participants also mentioned referrals by other patients to a psychologist or a psychiatrist as helpful encouragement toward helpseeking.

The actual practical support from friends and family is also seen to be an important help, even for individuals who have low intention to seek help. Depression patients often share a common symptom of hiding their emotions. Therefore, friends and family are considered to be the key people to help patients to realize their own illness and obtain help. This is most useful when the family and friends would actually involve and assist individuals to seek help. Such awareness by family and friends would require increase public health education on symptoms of depression and availability and approachability of help and treatments. Also, some participants shared accounts of a type of support that comes through popular online social networks. Through such networks, it is possible that depressive episodes can be more easily identified by their unpleasant effects. Family and friends might provide more preliminary encouragement and help, which in turn might delay the worsening process of the individual's depressive episodes.

\section{Treatment experience and subsequent help-seeking attitudes}

An important point that does not appear in the diagram is that previous treatment experience is also a key factor for individuals in determining whether to seek help or not. The views from participants who have experienced depression indeed differ greatly from those who have not, because the former speak from actual experience and the latter speak from their own attitudes and beliefs. However, we are able to see that previous treatment experience has greatly affected the former patients in terms of their subsequent help-seeking intentions and preferences. This is strongly linked to the perceived effectiveness of the treatment they have experienced. One participant who experienced depression indicated that drug use is his preferred treatment, and has been helping him through his depression. On the contrary, two other participants who had suffered from depression said that in their cases drug use was ineffective and had made them drowsy and prevented them from thinking effectively. They said their preference leaned toward nonpharmacological options or "breaking through" by themselves. This might even apply to individuals who have not experienced depression when they see the ineffectiveness of treatment for their family or friends. One participant specifically expressed this concern about drug use, saying that she thought it did not help treat her mother even though she had taken drugs for years. From these findings, it can be concluded that previous treatment experience has a great influence on subsequent help-seeking intention in the future.

\section{Discussion}

Guided by the TPB, this study illustrates a mechanism by which various factors contribute to an individual's attitude and help-seeking behavior regarding depression. These insights are helpful with the design of mental health promotion campaigns or interventions. We have been able to identify several relevant cultural elements, such as the importance of family support and depression as a manifestation of severe stress in Hong Kong. Further, depressive episodes are seen as a result of failure to stress management of people with a weak personality.

\section{Implications for media campaign development}

The findings in this study can contribute to the gathering of more targeted information and creation of messages that could be used in designing a public health communication and preventive media campaign for the general public. The mass media have been used widely in helping to educate the public, making use of health information with potential to minimize stigma in the mental healthcare sector (Wahl 2003). Based on our findings, stigma is not the only obstacle toward help-seeking in Hong Kong. Instead, perceived view and support by the family and other factors might be more unique and influential to the individualistic and collectivist characteristics in Chinese society like Hong Kong (Rao et al. 2010). Future campaigns should then be redirected to other targets rather than narrowly focusing on stigma reduction. Media campaigns might be targeted not only toward individuals to encourage them to seek help if needed but also to promote awareness of depression and provision of support to individual through their social networks including friends and family members who might be going through depressive episodes. By refocusing the media messages in the depression help- 
seeking campaigns, it is hoped that more targeted information can reach the public and encourage help-seeking and mutual support in the society. Also, theory-guided campaigns can also help to effectively encourage helpseeking by changes in beliefs and attitudes like suggested by the TPB (Schomerus et al. 2009).

\section{Support and awareness of social networks}

In our study, one uncertain factor that emerged is the unpredictable physical state of individuals at the point of depression. When one is depressed, one would be reluctant to engage in help-seeking and might not even realize one's own depressive symptoms (Griffiths et al. 2011a), no matter how positive one's attitude toward help-seeking might be under healthy conditions. This might be seen as a major limitation in that individuals could not accurately predict how they would react when they are indeed experiencing depression (Barney et al. 2006; Sherwood et al. 2007). However, our findings suggest that participants find it easier to identify depressive episodes in their family and friends instead of themselves. As for preference of treatment, a recent study also found adolescents' tendency to recommend a friend than themselves to seek help from a psychologist (Raviv et al. 2009). That insight suggests an alternative approach to promoting help-seeking that has in fact been suggested by previous research and campaigns. This approach emphasizes support from social networks and draws upon recommendations from others as essential tools to encourage help-seeking (Dew et al. 1988; Van Hook 1999; Lindsey et al. 2006). Family has been found to be a crucial unit to help prevent and cope with depression and provide emotional and informational support (Cabassa 2007; Ajzen 1986; Lawrence et al. 2006; Okello and Neema 2007; Griffiths et al. 2011b). However, strengthening support from social networks is rarely a major target of public health communication media campaigns for mental health promotion (Griffiths et al. 2011b).

This suggests that it may be essential for the public to be informed about how to monitor and offer help at an early stage to friends and family members who might be experiencing depression. However, research has shown that support from family and friends is positive and essential but can also bring disadvantages including stigma or inappropriate support (Griffiths et al. 2011b) Therefore, this campaign information should include the symptoms of depression and treatment options as well as the importance of support from family and friends for individuals confronting depression. Though studies find that some patients might be less likely or willing to seek help from family and friends when they experience depression, it is still important to educate the public on what those individuals may be experiencing and the importance of offering help (Jorm et al. 2000b; van Wijngaarden et al.
2004). There is no easy way to alter the common reluctance of those suffering with depression to seek help, and therefore it is important to build awareness and support for both individuals and their social networks to encourage help-seeking behavior (Frojd et al. 2007).

\section{Holistic approach to mental health education}

Our findings suggest that, despite an increasing awareness of clinical depression in the society, many members of the public have insufficient knowledge about the symptoms, etiology, treatment options, and prognosis of depression to enable them to make informed decisions on help-seeking or encouraging others to seek help. We can see that the public has a general understanding about some of the basic characteristics and clinical symptoms of depression, but many have not been exposed to a holistic view toward the choice of treatments, their efficiency, function, price and accessibility. At the same time, some may have concerns about treatment options, including privacy, accessibility, affordability, long-waiting times and efficiency. All of these factors come together to present a hierarchy of barriers toward individual decision-making about help-seeking (Jorm et al. 2000b). It has been suggested that mental health literacy is an important determinant of help-seeking (Jorm et al. 2000b). Therefore, it is important for health campaigns to provide precise educational data that speak to the above concerns in order to improve mental health literacy, reduce stigma encourage help-seeking (Gulliver et al. 2010). This campaign information should include the identification of depressive symptoms, characteristics and knowledge about mental health service providers (Gulliver et al. 2010). Specifically, information for the public should not be limited to pharmacological treatments but also should cover other non-pharmacological and evidence-based options including cognitive behavioral therapy, taking part in sports or even music therapy (Erkkilä et al. 2011; Goldney 1998). The pricing and accessibility of the full range of treatments should be listed in order to make campaign materials informative and user-friendly.

Participants who have experienced depression and treatment seem to be more aware of the negative consequence of delaying a decision to receive treatment. On the other hand, many participants who have not experienced depression mentioned delaying treatment decisions until depression symptoms become severe. In order to help the public to understand the significance of early decisions about seek help, campaign materials may include recommendations from people who have experienced depression (Dew et al. 1988). These examples could illustrate ways to seek help and promote the importance of seeking help (Lawrence et al. 2006). Our findings also suggest the importance of disseminating knowledge of various treatment options. It might therefore be an effective strategy to 
promote evidence-based and alternative treatments like bibliotherapy, music therapy or doing sports, which have been found to help relieve depression before symptoms become serious (Erkkilä et al. 2011). Sherwood et al. (2007) found that early help-seeking is essential in facilitating treatment of depression (Sherwood et al. 2007). With more knowledge and sharing from people who have experienced depression, the public may be encouraged to seek help earlier (Dew et al. 1988). Dietrich et al. (2010) also advocated that documenting how depression can happen to any individual, regardless of background, can pave the way to early treatment (Dietrich et al. 2010).

The limitation to this study is that the majority of participants are aged 20-25, which might not be able to portray the differences in factors related to incentive to help-seeking across different age groups in the public. Also, the sampling size might not be representative enough to illustrate the diversity in such a multi-cultural and densely populated city like Hong Kong. The factors around help-seeking and depression are also very complex and multi-faceted which need further research and investigation.

\section{Conclusions}

To conclude, more audience-oriented media campaigns for depression are greatly needed in order to encourage help-seeking behavior. It is hoped that this research on help-seeking incentive change would lead to more effective measures including media campaigns to encourage the public to seek help in the future. At policy level, it is also hoped that treatment resources and help can be made more accessible, affordable and effective so to attract public to seek help when they experience depression.

\section{Appendix 1}

Questions for all participants (Reference to MINI) (Groleau et al. 2006)

Do you think you know about depression?

Do you know anyone with depression?

What will come to your mind immediately when you think about depression?

Would you call depression a disease?

If you have to differentiate unhappiness from depression, how different is it?

How long does depression last?

Would you say unhappiness is more like the preliminary stage of depression?

What makes unhappiness become depression?

What do you think is the cause of depression?

For people you know that have experienced depression, do you really see biological factors?

What are the available treatments you can think of?

What do you think people around you think about depression in Hong Kong?
For someone very close to you, what symptoms would make you think he/she has depression?

For how long would you observe until you raise the issue?

At what point do you think there should be extra help?

What would you do in practical terms to help?

When would such a person need to see a psychiatrist?

If the counselor suggests that a person such as your boyfriend, for example, visit a psychologist or psychiatrist, do you think that person would agree to go?

For psychologists and psychiatrists, do you know what treatment they provide?

What is your preference between different treatments? Do you think you would actively seek help for yourself?

Do you think people consider help-seeking to be a bad thing?

How would you help people to view help-seeking as something not bad?

Do you think people tend to link depression to weakness?

What is the efficacy of medicine?

Do you think depression is curable?

If your family were not supportive of you seeking help, would you still seek help?

What do you think the public should know about depression?

How do you convince a person that depression is not a bad thing?

If they really need external help, how are you going to phrase it?

How have the media described depression?

\section{Extra questions for participants with depression experience}

When did you experience depression?

When you did you realize you had this health problem? What happened when you had this illness?

Did you seek help from a doctor? How was the visit to the doctor or hospital?

Did you receive any tests or treatment?

Has anyone at work or school experienced depression?

Have you ever seen in any media that there are people who have the same health problem as yours?

In what way is that person's problem similar to or different from yours?

How would you describe your health problem using other terms or expressions?

According to you, what caused your health problem?

Why did your health problem start when it did?

Is there something happening in your family or at work or in your social life that could explain your health problem?

What does "depression" mean to you?

What usually happens to people who have depression? 
What is the best treatment for people who have depression?

How do other people react to someone who experiences depression?

Did your doctor/healer give you any treatment, medicine or recommendations to follow?

Are you able to follow that treatment?

What made that treatment work well?

What has helped you through this period in your life?

How have your family or friends helped you through

this difficult period of your life?

How has your spiritual life, faith or religious practice helped you go through this difficult period of your life?

Did you want to seek help from a doctor/healer?

What did your family think about you seeking help?

What did your friends think about you seeking help?

Who would be supportive of or opposed to your helpseeking?

What do you think about the action of help-seeking?

Is it easy or difficult for you to seek help?

\section{Competing interests}

The authors declare that they have no competing interests.

\section{Authors' contributions}

KWF developed the grant proposal and the overall research idea and obtained the grant. KWF and PW designed the methodology; AH conducted the interviews and collected the data; KWF and AH performed the analysis; AH wrote the first draft of the manuscript; all authors revised the manuscript for important intellectual content and approved the final version to be submitted for publication.

\section{Acknowledgements}

This study and AH's Postgraduate Scholarship are supported by the General Research Fund, Research Grants Council, Hong Kong (HKU 744410H). The writing of this article was partly funded by the approval of sabbatical leave to PWCW granted by the Faculty Human Resource Committee (FHRC) of the Faculty of Social Sciences, The University of Hong Kong.

\section{Author details}

'Journalism and Media Studies Centre, The University of Hong Kong, Hong Kong, China. ${ }^{2}$ Department of Social Work and Social Administration, The University of Hong Kong, Hong Kong, China.

\section{Received: 5 February 2013 Accepted: 25 March 2014}

Published: 7 April 2014

\section{References}

Ajzen, I. (1986). Dispositional prediction of behavior in personality and socialpsychology. Bull Br Psychol Soc, 39, A65-A65.

Ajzen, I. (1991). The theory of planned behavior. Organ Behav Hum Decis Process, $50(2), 179-211$

Barney, LJ, Griffiths, KM, Jorm, AF, \& Christensen, H. (2006). Stigma about depression and its impact on help-seeking intentions. Aust N Z J Psychiatry, $40(1), 51-54$

Barney, L, Griffiths, KM, Christensen, H, \& Jorm, AF. (2009). Exploring the nature of stigmatising beliefs about depression and help-seeking: Implications for reducing stigma. BMC Public Health, 9, 61 .

Ben-Porath, DD. (2002). Stigmatization of individuals who receive psychotherapy: An interaction between help-seeking behavior and the presence of depression. J Soc Clin Psychol, 21(4), 400-413.

Blay, SL, Furtado, A, \& Peluso, ETP. (2008). Knowledge and beliefs about helpseeking behavior and helpfulness of interventions for Alzheimer's disease. Aging Mental Health, 12(5), 577-586.
Burns, JR, \& Rapee, RM. (2006). Adolescent mental health literacy: young people's knowledge of depression and help seeking. J Adolesc, 29(2), 225-239.

Cabassa, LJ. (2007). Latino immigrant men's perceptions of depression and attitudes toward help seeking. Hisp J Behav Sci, 29(4), 492-509.

Chan, B. (2007). Depression through Chinese eyes: A Window into Public Mental Health in Multicultural Australia. Sydney, Australia: School of Public Health and Community Medicine and School of Psychiatry, University of New South Wales.

Cheung, CK, Leung, KK, Chan, WT, \& Ma, K. (1998). Depression, loneliness, and health in an adverse living environment: A study of bedspace residents in Hong Kong. Soc Behav Person, 26(2), 151-169.

Dew, MA, Dunn, LO, Bromet, EJ, \& Schulberg, HC. (1988). Factors affecting help-seeking during depression in a community sample. J Affect Disord 14(3), 223-234.

Dietrich, S, Mergl, R, Freudenberg, P, Althaus, D, \& Hegerl, U. (2010). Impact of a campaign on the public's attitudes towards depression. Health Educ Res, 25(1), 135-150.

Ebigbo, PO. (1982). Development of a culture specific (Nigeria) screening scale of somatic complaints indicating psychiatric disturbance. Cult Med Psychiatry, 6(1), 29-43.

Eisenhardt, KM. (1989). Building theories from case study research. Acad Manag Rev, 14(4), 532-550.

Elder, RW, Shults, RA, Sleet, DA, Nichols, JL, Thompson, RS, \& Rajab, W. (2004). Effectiveness of mass media campaigns for reducing drinking and driving and alcohol-involved crashes: a systematic review. Am J Prev Med, 27(1), 57-65.

Erkkilä, J, Punkanen, M, Fachner, J, Ala-Ruona, E, Pöntiö, I, Tervaniemi, M, \& Gold, C. (2011). Individual music therapy for depression: randomised controlled trial. Br J Psychiatry, 199(2), 132-139.

Frojd, S, Mauri, M, Mirjami, P, von der Pahlen, B, \& Kaltiala-Heino, R. (2007). Adult and peer involvement in help-seeking for depression in adolescent population. Soc Psychiatry Psychiatr Epidemiol, 42(12), 945-952.

Glaser, BG, \& Strauss, AL. (1967). The Discovery of Grounded Theory: Strategies for Qualitative Research. Chicago: Aldine de Gruyter.

Goldney, RD. (1998). Suicide prevention is possible: a review of recent studies. Arch Suicide Res, 4(4), 329-339.

Good, GE, \& Wood, PK. (1995). Male gender-role conflict, depression, and help-seeking - Do college men face double jeopardy. J Couns Dev, 74(1), 70-75.

Grausgruber, A, Schöny, W, Grausgruber-Berner, R, Koren, G, Apor, BF, Wancata, J, \& Meise, U. (2009). "Schizophrenia has many faces"-evaluation of the Austrian anti-sigma-campaign 2000-2002. Psychiatr Prax, 36(07), 327-333.

Griffiths, KM, Crisp, DA, Jorm, AF, \& Christensen, H. (2011a). Does stigma predict a belief in dealing with depression alone? J Affect Disord, 132(3), 413-417.

Griffiths, KM, Crisp, DA, Barney, L, \& Reid, R. (2011b). Seeking help for depression from family and friends: a qualitative analysis of perceived advantages and disadvantages. BMC Psychiatry, 11, 196

Groleau, D, Young, A, \& Kirmayer, L. (2006). The McGill Illness Narrative Interview (MINI): an interview schedule to elicit meanings and modes of reasoning related to illness experience. Transcult Psychiatry, 43(4), 671-91.

Gulliver, A, Griffiths, KM, \& Christensen, H. (2010). Perceived barriers and facilitators to mental health help-seeking in young people: a systematic review. BMC Psychiatry, 10, 113

Halgin, RP, Weaver, DD, Edell, WS, \& Spencer, PG. (1987). Relation of depression and help-seeking history to attitudes toward seeking professional psychological help. J Couns Psychol, 34(2), 177-185.

Han, DY, Chen, SH, Hwang, KK, \& Wei, HL. (2006). Effects of psychoeducation for depression on help-seeking willingness: Biological attribution versus destigmatization. Psychiatry Clin Neurosci, 60(6), 662-668.

Hegerl, U, Althaus, D, \& Stefanek, J. (2003). Public attitudes towards treatment of depression: effects of an information campaign. Pharmacopsychiatry, 36(6), 288-291.

Jorm, AF. (2000). Mental health literacy - Public knowledge and beliefs about mental disorders. Br J Psychiatry, 177, 396-401.

Jorm, AF, Korten, AE, Jacomb, PA, Christensen, H, Rodgers, B, \& Pollitt, P. (1997). "Mental health literacy": A survey of the public's ability to recognise mental disorders and their beliefs about the effectiveness of treatment. Med J Aust, 166(4), 182-186.

Jorm, AF, Angermeyer, M, \& Katschnig, H. (2000a). Public knowledge of and attitudes to mental disorders: a limiting factor in the optimal use of treatment services. Unmet Need Psychiatry, 413, 399-413. 
Jorm, AF, Christensen, H, Medway, J, Korten, AE, Jacomb, PA, \& Rodgers, B. (2000b). Public belief systems about the helpfulness of interventions for depression: associations with history of depression and professional help-seeking. Soc Psychiatry Psychiatr Epidemiol, 35(5), 211-219.

Jorm, AF, Allen, NB, O'Donnell, CP, Parslow, RA, Purcell, R, \& Morgan, AJ. (2006) Effectiveness of complementary and self-help treatments for depression in children and adolescents. Med J Aust, 185(7), 368-72.

Kleinman, A. (1988). The Illness Narratives: Suffering, Healing, and the Human Condition. New York: Basic Books.

Kleinman, A. (2004). Culture and depression. N Engl J Med, 351(10), 951-953.

Kleinman, A, Anderson, J, Finkler, K, Frankenberg, R, \& Young, A. (1986). Social origins of distress and disease: Depression, neurasthenia, and pain in modern China. Curr Anthropol, 24(5), 499-509.

Lawrence, V, Banerjee, S, Bhugra, D, Sangha, K, Turner, S, \& Murray, J. (2006). Coping with depression in later life: a qualitative study of help-seeking in three ethnic groups. Psychol Med, 36(10), 1375-1383.

Lee, S. (1999). Mental health problems in transition: challenges for psychiatry in Hong Kong. Hong Kong Med J, 5(1), 6.

Lee, DTS, Kleinman, J, \& Kleinman, A. (2007). Rethinking depression: An ethnographic study of the experiences of depression among Chinese. Harvard Rev Psychiatry, 15(1), 1-8.

Lindsey, MA, Korr, WS, Broitman, M, Bone, L, Green, A, \& Leaf, PJ. (2006). Helpseeking behaviors and depression among African American adolescent boys. Soc Work, 51(1), 49-58.

Mo, P, \& Mak, W. (2008). Using an extended theory of planned behavior to understand help-seeking for mental health problems among Chinese. Int J Psychol, 43(3-4), 571-571.

Murray, CJL, Vos, T, Lozano, R, Naghavi, M, Flaxman, AD, Michaud, C, \& Lopez, AD. (2012). Disability-adjusted life years (DALYs) for 291 diseases and injuries in 21 regions, 1990-2010: a systematic analysis for the Global Burden of Disease Study 2010. Lancet, 380(9859), 2197-2223.

Okello, ES, \& Neema, S. (2007). Explanatory models and help-seeking behavior: pathways to psychiatric care among patients admitted for depression in Mulago Hospital, Kampala. Uganda. Qual Health Res, $17(1), 14-25$.

Patton, MQ. (2002). Qualitative Research and Evaluation Methods. Thousand Oaks, California: Sage Publications, Inc.

Paykel, ES, Hart, D, \& Priest, RG. (1998). Changes in public attitudes to depression during the Defeat Depression Campaign. Br J Psychiatry, 173, 519-522.

Rao, D, Horton, RA, Tsang, HW, Shi, K, \& Corrigan, PW. (2010). Does individualism help explain differences in employers' stigmatizing attitudes toward disability across Chinese and American cities? Rehabil Psychol, 55(4), 351.

Raviv, A, Raviv, A, Vago-Gefen, I, \& Fink, AS. (2009). The personal service gap: factors affecting adolescents' willingness to seek help. J Adolesc, 32(3), 483-499.

Reiling, DM. (2002). Boundary maintenance as a barrier to mental health helpseeking for depression among the Old Order Amish. J Rural Health, 18(3), $428-436$.

Rice, RE, \& Atkin, CK. (2013). Public Communication Campaigns. Thousand Oaks, California: Sage Publications, Incorporated.

Roness, A, Mykletun, A, \& Dahl, AA. (2005a). Help-seeking behaviour in patients with anxiety disorder and depression. Eur Psychiatry, 20, S126-S127.

Roness, A, Mykletun, A, \& Dahl, AA. (2005b). Help-seeking behaviour in patients with anxiety disorder and depression. Acta Psychiatr Scand, $111(1), 51-58$.

Salloum, IM, \& Mezzich, JE. (2009). Psychiatric Diagnosis: Challenges and Prospects (Vol. 8). Chichester, UK ; Hoboken, NJ: Wiley.

Schomerus, G, Matschinger, H, \& Angermeyer, M. (2009). Attitudes that determine willingness to seek psychiatric help for depression: a representative population survey applying the Theory of Planned Behaviour. Psychol Med, 39(11), 1855.

Sherwood, C, Salkovskis, PM, \& Rimes, KA. (2007). Help-seeking for depression: The role of beliefs, attitudes and mood. Behav Cogn Psychother, 35(5), 541-554.

Van Hook, MP. (1999). Women's help-seeking patterns for depression. Soc Work Health Care, 29(1), 15-34.

van Wijngaarden, B, Schene, AH, \& Koeter, MWJ. (2004). Family caregiving in depression: impact on caregivers' daily life, distress, and help seeking. J Affect Disord, 81(3), 211-222.

Wahl, OF. (2003). News media portrayal of mental illness - Implications for public policy. Am Behav Sci, 46(12), 1594-1600.
Wakefield, MA, Loken, B, \& Hornik, RC. (2010). Use of mass media campaigns to change health behaviour. Lancet, 376(9748), 1261-1271.

Wallace, WL. (1971). The Logic of Science in Sociology. New York: Transaction Publishers.

WHO. (2011). WHO Depression Fact Sheet. Genève: World Health Organization. Available from: http://www.who.int/mediacentre/factsheets/fs369/en/ Accessed 10 April 2014.

doi:10.1186/2050-7283-2-9

Cite this article as: Hui et al.: Building a model for encouraging helpseeking for depression: a qualitative study in a Chinese society. BMC Psychology 2014 2:9.

\section{Submit your next manuscript to BioMed Central and take full advantage of:}

- Convenient online submission

- Thorough peer review

- No space constraints or color figure charges

- Immediate publication on acceptance

- Inclusion in PubMed, CAS, Scopus and Google Scholar

- Research which is freely available for redistribution 